\title{
A Circuit for Coding Interaural Time Differences in the Chick Brainstem
}

\author{
Edwin M. Overholt, Edwin W Rubel, and Richard L. Hyson \\ Hearing Development Laboratories, Departments of Otolaryngology-Head and Neck Surgery, and Physiology and \\ Biophysics, University of Washington, Seattle, Washington 98195
}

Third-order auditory neurons in the avian nucleus laminaris (NL) are the first to receive binaural input. In the chick, NL consists of a monolayer of neurons with polarized dendritic arbors oriented dorsally and ventrally. Afferents from second-order neurons in the ipsilateral nucleus magnocellularis (NM) innervate the dorsal dendrites of NL neurons, distributing processes of approximately equal length to NL neurons along an isofrequency band (roughly caudomedial to rostrolateral). Afferents from the contralateral NM innervate the ventral dendrites of NL neurons, distributing collateral branches sequentially as they proceed from caudomedial to rostrolateral along the Isofrequency band of neurons. This innervation pattern could be the basis of a "delay line" circuit, as postulated in models of neural networks mediating sound localization.

We examined this circuit by analyzing evoked field potentials using a brain slice preparation containing both NL and NM. The results were consistent with the previous anatomical findings. When the ipsilateral auditory nerve or ipsilateral NM was stimulated, there was no consistent variation in the latency of postsynaptic field potentials across the medial-to-lateral extent of NL. In contrast, when the contralateral NM or NM axons in the crossed dorsal cochlear tract were stimulated, a linear increase in the latency of postsynaptic potentials was observed from medial to lateral positions in NL.

When stimulation amplitudes for both the ipsilateral and contralateral inputs were adjusted so as to produce little or no postsynaptic field potential, simultaneous bilateral stimulation evoked a pronounced response. Thus, NL neurons can act as "coincidence detectors." The amplitude of the postsynaptic response was dependent on the relative timing of stimulation of the two inputs. The optimal time difference changed systematically across the medial-to-lateral extent of NL. This system of delay lines and coincidence detectors could provide a mechanism for converting interaural time differences into a "place map" within NL.

\footnotetext{
Received Aug. 23, 1991; revised Nov. 25, 1991; accepted Dec. 2, 1991.

This work was supported by U.S. Public Health Service Grants DC 00395, DC 00018 , and DC 00858. We thank C. Carr, L. Kitzes, M. Konishi, T. Yin, E. Lachica, D. Mills, A. Reyes, A. Joseph, and W. E. Sullivan for their comments on carlicr versions of the manuscript.

Correspondence should be addressed to Richard L. Hyson, Ph.D., Hearing Development Laboratories, Department of Otolaryngology-Head and Neck Surgery, RL-30, University of Washington, Seattle, WA 98195.

Copyright (C) 1992 Society for Neuroscience $0270-6474 / 92 / 121698-11 \$ 05.00 / 0$
}

Binaural hearing is critical for signal detection and localization of a sound source in space. The two major binaural cues involved in sound localization are interaural time (or phase) differences and interaural intensity differences. As postulated in the classic duplex theory (Stevens and Newman, 1936), the interaural difference in sound level, resulting from the acoustic shadow cast by the head, is the predominate cue for localization of high-frequency sounds. In contrast, the principal binaural cue for localization of low-frequency sounds or sound envelopes is the difference in the timing (or phase) of acoustic information as sound waves traverse the head from one ear to the other.

Several models of binaural signal processing include a nucleus within the brainstem that encodes information relating to interaural time disparities (Jeffress, 1948; von Békésy, 1960; van Bergeijk, 1962; Colburn and Durlach, 1978). In the classic model of Jeffress (1948), an interaural time difference is represented as a "place" of coincident binaural activation. This model, as diagrammed in Figure $1 A$, consists of a series of "delay lines" that project onto an array of neurons. Transmission delay is indicated by the length of the lines on each side of the array of cells. The postsynaptic neurons function as "coincidence detectors" that increase their firing rate when inputs arrive at both sides of the soma simultaneously. In this model, each represented interaural time disparity would produce simultaneous bilateral stimulation of a specific subset of the neurons. This combination of delay lines and coincidence detectors forms the basis of a "place" theory of sound localization.

Delay lines. A topographic arrangement of auditory projections that resembles delay lines such as those postulated in Jeffress's (1948) model was first described in the chicken brainstem (Young and Rubel, 1983). Auditory nerve fibers project from the periphery to nucleus magnocellularis (NM) (Boord, 1969; Rubel and Parks, 1975). As in mammals (Rose et al., 1967), the auditory nerve input to the cochlear nucleus (NM) is phase-locked to the acoustic stimulus (Rubel and Parks, 1975; Sullivan and Konishi, 1984; Warchol and Dallos, 1989, 1990). Each NM neuron projects bilaterally to nucleus laminaris (NL) (Hackett et al., 1982; Young and Rubel, 1983), which is similar to the medial superior olivary nucleus in mammals. In the chick, NL consists of a monolayer of cells that have symmetric dendritic arbors oriented into distinct dorsal and ventral fields (Smith and Rubel, 1979). Afferents from the ipsilateral NM innervate the dorsal dendrites of NL, while the contralateral NM sends axons, via the crossed dorsal cochlear tract, to the ventral dendrites (Boord, 1969; Parks and Rubel, 1975). Both the ipsilateral and contralateral projections from an individual NM neuron innervate an isofrequency line of cells (oriented roughly cau- 

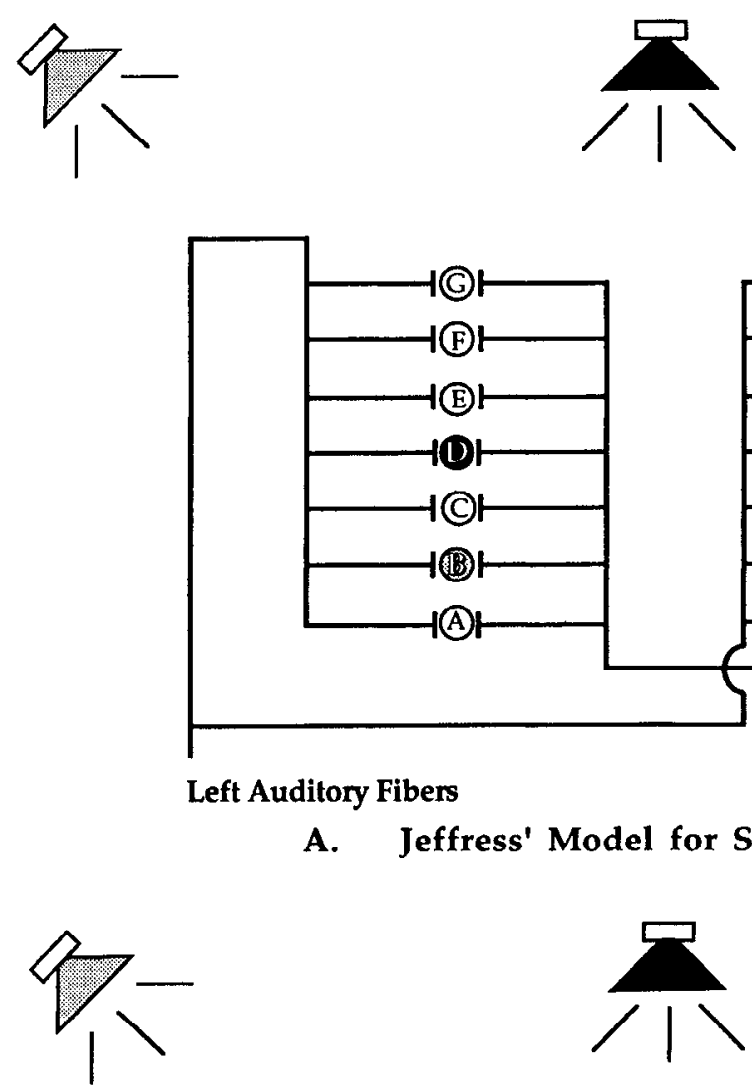

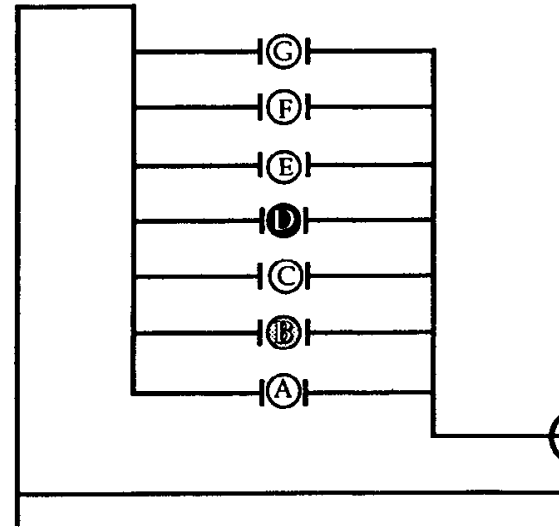

Left Auditory Fibers

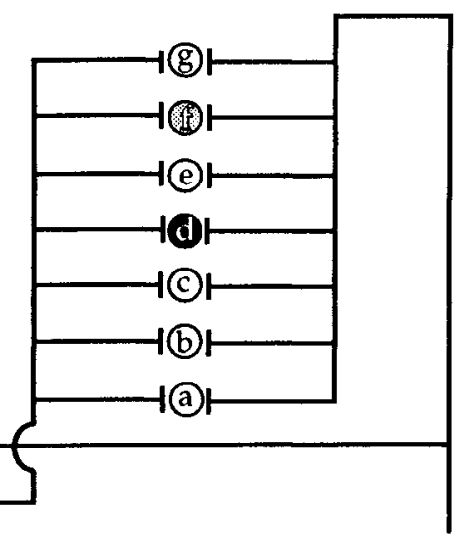

Right Auditory Fibers

A. Jeffress' Model for Sound Localization
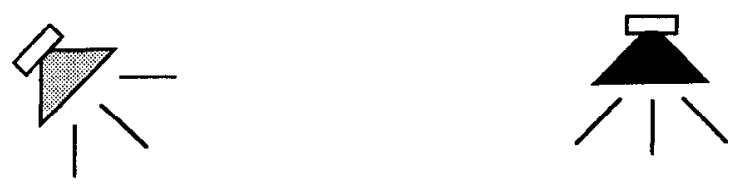

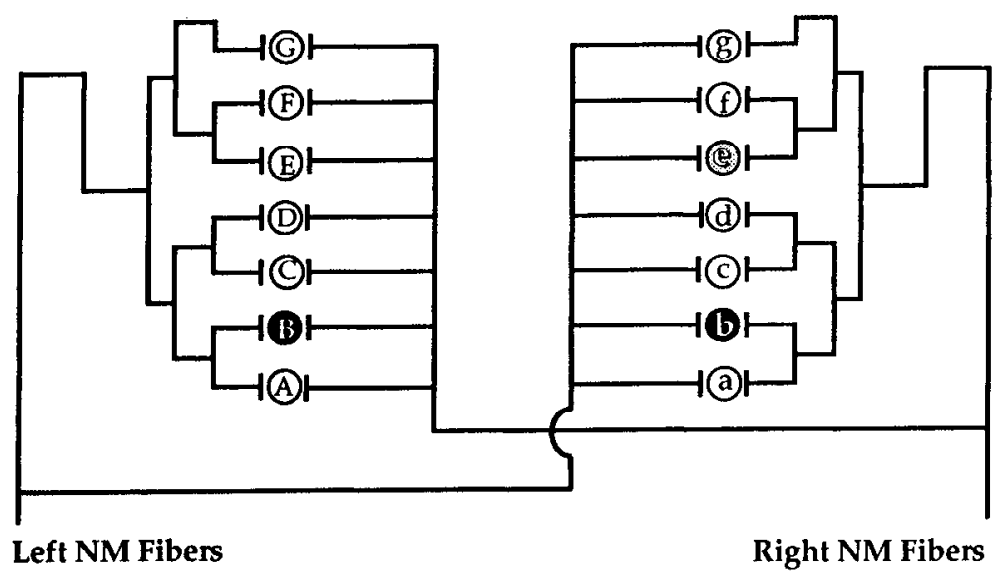

B. Nucleus Laminaris Model for Sound Localization
Figure 1. A, Jeffress's (1948) model of sound localization. When a sound source is located at midline, neurons in the middle of an array of cells (cells $D$ and $d$ ) will receive input from the right and left side simultaneously. When the sound source is located off to one side, impulses along fibers on the near side will travel farther and arrive simultaneously with impulses from the other side at a more distant cell (e.g., cells $B$ and $f$ ). $B$, Modification of Jeffress's model incorporating features of $\mathrm{NL}$ of the chick. In this modification, the "delay line" is strictly in the contralateral projection and the NL on each side of the brain primarily encodes time delays from sounds originating in the contralateral hemifield. domedial to rostrolateral) along NL (Young and Rubel, 1983, 1986). The terminal branching patterns of the ipsilateral and contralateral projections to this line of cells, however, differ. Each axon from the ipsilateral NM appears to project to an isofrequency band of NL neurons with processes of roughly equivalent length. In contrast, an axon from the contralateral NM makes synapses sequentially along this band of cells such that neurons in the medial portion of NL have shorter afferents from the contralateral NM than neurons located in the lateral aspect of NL (see Fig. $1 B$ ). This arborization pattern of the axons arising from the contralateral NM could constitute a "delay line" for auditory information as hypothesized in Jeffress's model.

Delay lines have also been described in the barn owl both anatomically and physiologically. In contrast to the monolayer structure seen in the chicken, NL in the barn owl is expanded in the coronal plane. Consequently, in the chicken about 1000 neurons make up NL on each side of the brainstem (Rubel et al., 1976), whereas in the barn owl approximately 5000 neurons form this nucleus on each side (Winter and Schwartzkopff, 1961). The primary branching of incoming NM fibers in the barn owl resembles the innervation patterns of the chicken, but both ipsilateral and contralateral fibers further progress in the dorsoventral plane (Carr and Konishi, 1988, 1990). Physiological recordings in vivo suggest that delay lines in the barn owl exist in both the ipsilateral and contralateral projections to $\mathrm{NL}$ and are oriented in the dorsal-ventral plane (Sullivan and Konishi, 1986). These conclusions were based on recordings of a field potential known as the "neurophonic" evoked by acoustic stim- 
ulation. For the purpose of examining delay lines, this approach has two limitations. First, it is not clear what aspect of this response is presynaptic as opposed to postsynaptic or whether delays are duc to propertics of the presynaptic or the postsynaptic elements. Second, measurements of time delays across a nucleus requires that all inputs are activated synchronously. In vivo, this requires the assumption that the activity of all NM inputs to NL are locked to the same phase of the acoustic stimulus.

In the present experiments, we use an in vitro slice preparation of the chick brainstem auditory system to analyze the potential delay lines from NM across NL. In this preparation, presynaptic and postsynaptic response components are readily distinguishable and the timing of activation of the NM afferents to NL is accurately controlled. Based on the anatomy of this system, one can make a strong prediction regarding the timing of postsynaptic activation of NL neurons: stimuli arriving from the ipsilateral NM should activate all neurons along an isofrequency line in NL at nearly identical times, while contralatcral stimuli should activate medial NL neurons first and subsequently more lateral neurons with a progressively increasing delay.

Coincidence detection. The second feature of Jeffress's (1948) model is that postsynaptic cells serve as coincidence detectors. Recordings from single units in the owl have shown that NL neurons do increase firing rate when inputs from the two ears arrive in phase (Carr and Konishi, 1990). Similarly, a number of studies have shown that the response of neurons in the medial superior olivary nucleus of mammals to binaural stimulation is dependent on the interaural time difference (Galambos et al., 1959; Moushegian et al., 1964, 1975; Goldberg and Brown, 1968, 1969; Guinan et al., 1972; Crow et al., 1978; Yin and Chan, 1988, 1990). It has not been conclusively demonstrated, however, that optimal interaural time differences are systematically mapped across this nucleus. In NL of the chick, input from the contralateral NM should arrive at medial portions of the nucleus prior to arriving at lateral portions of the nucleus. Thus, the contralateral lead time required to evoke the maximal response to bilateral stimulation should vary systematically across the medial-to-lateral extent of the nucleus. Specifically, longer contralateral lead times should be required to evoke the maximal response at more lateral locations in NL. The present experiments provide further evidence for coincidence detection by NL neurons in the chick and demonstrate that optimal time differences vary systematically across NL.

\section{Materials and Methods}

\section{Preparation and maintenance of slices}

White Leghorn chickens $(H+N$, Redmond, WA) were obtained as 1-dold hatchlings or hatched from eggs incubated in the University of Washington vivarium. The chickens were raised in communal brooders with unrestricted access to food and water. All brainstem slices used in this study were obtained from 5-15 d posthatch chicks. The subjects were decapitated and the brainstems were rapidly removed while immersed in oxygenated artificial cerebrospinal fluid (ACSF) at room temperature. The brainstem was attached to the stage of a vibratome using cyanoacrylate glue, and gelatin ( $25 \%$ in ACSF) was injected under the lateral aspects of the brainstem for additional support. Coronal brainstem slices $400 \mu \mathrm{m}$ thick containing NM and NL were prepared and transferred to a recording chamber modeled after that used by Oertel (Oertel, 1983, 1985; Hyson and Rubel, 1989). The slice was placed on a small piece of lens paper and stabilized with an overlying cotton mesh gauze that was secured with metal clips. The slices were perfused continuously with warm, oxygenated $\left(95 \% \mathrm{O}_{2}, 5 \% \mathrm{CO}_{2}\right)$ ACSF at a rate of $3 \mathrm{ml} / \mathrm{min}$. Normal ACSF consisted of (in $\mathrm{mM}$ ) NaCl, 130; KCl, 3.0; $\mathrm{NaHCO}_{3}, 26 ; \mathrm{MgSO}_{4}, 2.0 ; \mathrm{CaCl}_{2}, 2.0 ; \mathrm{NaH}_{2} \mathrm{PO}_{4}, 1.25 ;$ and dextrose, 10
(pH 7.3). For low-calcium/high-magnesium perfusate the composition was identical with the exception that $0.5 \mathrm{~mm} \mathrm{CaCl}$ and $5 \mathrm{~mm} \mathrm{MgSO}_{4}$ were used. The temperature of the perfusate in the chamber was maintained at $34-35^{\circ} \mathrm{C}$ except where noted otherwise. The slices were allowed to equilibrate for at least $30 \mathrm{~min}$ prior to data collection.

\section{Electrophysiological procedures}

General procedures. Bipolar stimulating electrodes were constructed from two twisted strands of Teflon-coated silver wire. NM and NL could be easily identified under low-power magnification with a stereomicroscope. General procedures are outlined in Figure 2, $A$ and $C$. Ipsilateral orthodromic activation of NL neurons was achieved by placing a bipolar electrode on either the ipsilateral NM or auditory nerve (VIII n.). Contralateral stimulation of NL neurons was achieved by placing another bipolar electrode on the fibers of the crossed dorsal cochlear tract or on the contralateral NM. In all cases, a square wave stimulus of $20 \mu \mathrm{sec}$ duration was used with an interstimulus interval of $300 \mathrm{msec}$. Stimulation voltage varied between slices from 5 to $50 \mathrm{~V}$ but remained constant within each slice during the recording of evoked field potentials.

Micropipettes were made from thin-wall (1 $\mathrm{mm}$ o.d.) glass tubing, filled with $4 \mathrm{M} \mathrm{NaCl}$ and broken to a resistance between 1 and $3 \mathrm{M} \Omega$. Fast green (1\%) was added to aid visualization of the electrode tip. The micropipettes were lowered onto NL using a three-dimensional micromanipulator. Movement of the micromanipulator was calibrated using a stage micrometer to allow for recording of electrode position in Cartesian coordinates. These coordinates were used to calculate the relative recording positions within NL. The potentials were amplified and recorded using a multichannel FM tape recorder. Off-line analysis and signal averaging were performed using a digital storage oscilloscope.

Delay lines. In all of the experiments reported here, the stimulating electrode was maintained in a fixed position while field potentials were recorded at various medial to lateral positions along NL. In four experiments, recordings were made simultaneously from separate positions within NL. In two slices, evoked potentials across the entire medial-to-lateral dimension of NL could be obtained by either ipsilateral or contralateral stimulation. More commonly, only ipsilateral stimulation $(n=3)$ or only contralateral stimulation $(n=4)$, but not both, would yield reliable postsynaptic potentials along the entire course of NL. The latencies of both the presynaptic (NM afferent fibers) and postsynaptic potentials were recorded as a function of the position of the recording electrode along the medial-to-lateral dimension of NL. The latency was measured from the trigger signal to the point of maximum deflection in the averaged field potential using the cursor of the digital storage oscilloscope.

The distance between each successive recording position was estimated from the Cartesian coordinates derived from the calibrated movements of the micromanipulator. After the evoked potential at a certain position was recorded, the recording electrode was raised and repositioned nearby within NL. Successive repositioning of the microclectrode along NL yielded a set of Cartesian coordinates for each recording position. Using these coordinates, the linear distance between each successive recording position was calculated by applying the Pythagorean theorem: $\left(\left(X_{1}-X_{2}\right)^{2}+\left(Y_{1}-Y_{2}\right)^{2}\right)^{1 / 2}$, where $\left(X_{1}, Y_{1}\right)$ and $\left(X_{2}\right.$, $\left.Y_{2}\right)$ are the coordinates of two adjacent recording positions. The entire length of the nucleus was calculated by summing the linear distances between each successive position. The correlation of the latency of the evoked potentials with the location along NI, was examined after either ipsilateral or contralateral stimulation. For simplicity, linear relations between distance and latency were assumed, although higher-order polynomial equations usually fitted the data slightly better.

Axon conduction velocities were calculated by measuring the latencies of the presynaptic field potentials during contralateral stimulation. A scatter plot of latency as a function of distance within NL was constructed and a least-squares regression performed. Conduction velocities of the afferent fibers were estimated from the slope of the regression lines ( $n=4$ slices). The variation of axon conduction velocity as a function of temperature was also examined in six slices. In these experiments, medial and lateral recording electrodes were positioned and left undisturbed while the temperature of the ACSF perfusate was varied from $29^{\circ} \mathrm{C}$ to $42^{\circ} \mathrm{C}$ at a rate of $1^{\circ} \mathrm{C}$ per $4 \mathrm{~min}$. The distance between the two recording electrodes in NL was calculated as described above.

Coincidence detection. For analyses of coincidence detection and the mapping of optimal time delays, slices $(n=3)$ were stimulated bilaterally and the time between stimulating the contralateral and ipsilateral inputs was varied. The stimulation amplitude to each side was adjusted so as 
A
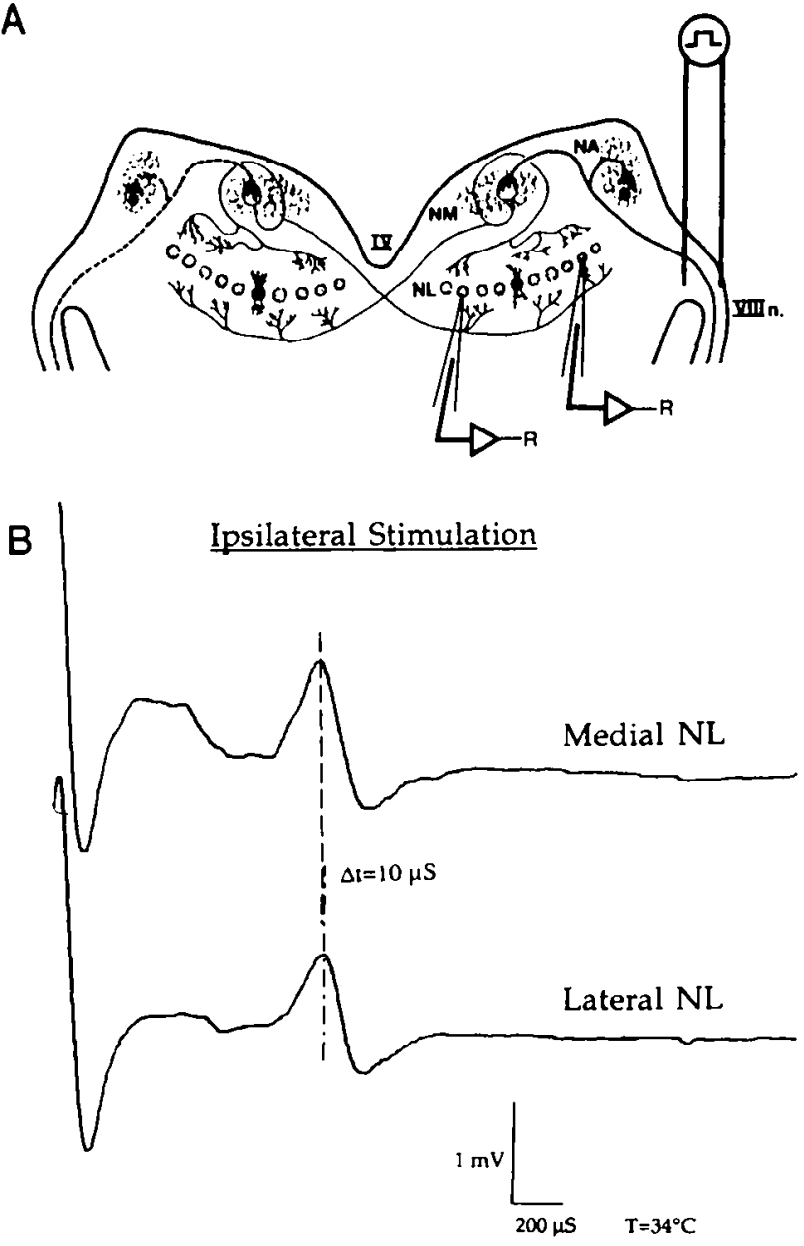

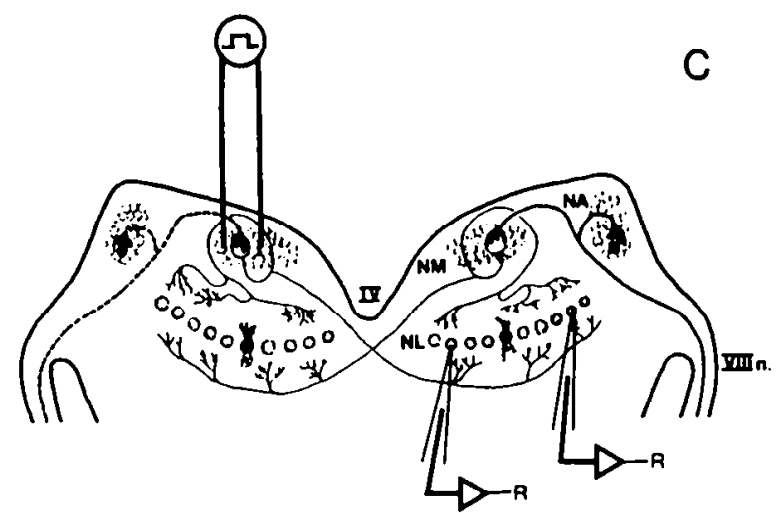

Contralateral Stimulation

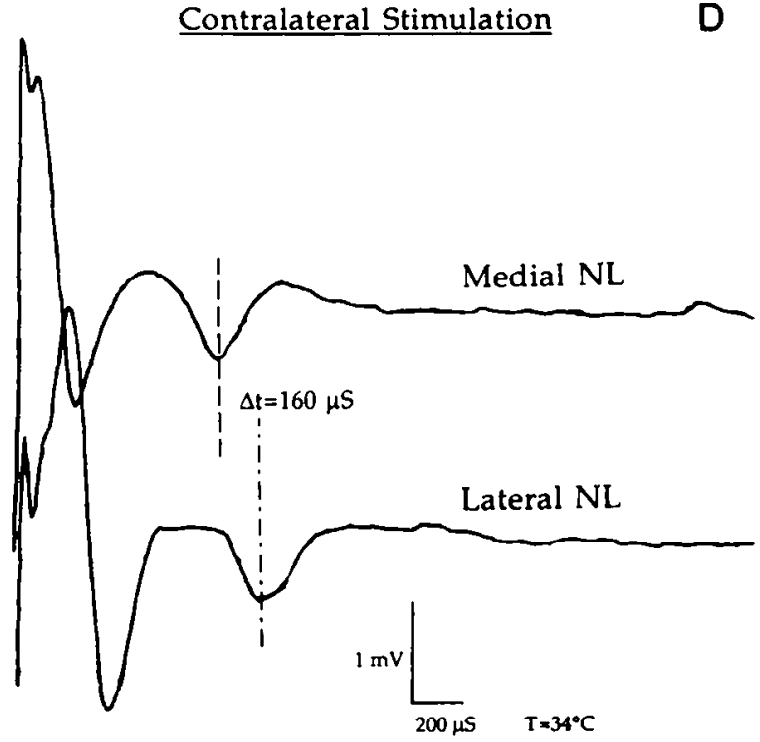

Figure 2. Schematic of the brainstem auditory system of the chick displaying the general methods. $A$, Ipsilateral stimulation: neurons in NL were stimulated orthodromically via activation of the auditory nerve, as depicted here, or by placing the stimulating electrode directly onto the ipsilateral NM. $C$, Contralateral stimulation: neurons in NL were stimulated orthodromically via activation of the contralateral NM, as depicted here, or by stimulating NM axons in the crossed dorsal cochlear tract. Recording electrodes $(R)$ were placed at different locations along the medial-to-lateral extent of NL and evoked potentials recorded. $B$ and $D$, Simultaneously recorded field potentials in medial and lateral NL following stimulation of the ipsilateral NM $(B)$ or stimulation of the axons from the contralateral NM in the crossed dorsal cochlear tract $(D)$. With ipsilateral stimulation, there is a minimal difference in the latency of the postsynaptic responses, whereas with contralateral stimulation, the latency of the postsynaptic response is consistently longer in the laterally placed recording electrode. $N A$, nucleus angularis.

to evoke little or no postsynaptic field potential in NL when presented alone. Observation of a reliable postsynaptic field potential when the slices were stimulated bilaterally was taken as evidence of coincidence detection. The amplitude of the postsynaptic field potential was measured as a function of the relative time between stimulating the two inputs. In one slice, mapping of optimal delay was demonstrated by recording field potentials simultaneously from two different positions along NL.

\section{Results}

\section{Field potentials}

Ipsilateral stimulation. Figures $2 B$ and 3 illustrate averaged ( $n$ $=8$ traces) field potentials recorded in NL during stimulation of the ipsilateral NM. Stimulation of the ipsilateral auditory nerve produced similar potentials. When the slice was perfused with low-calcium/high-magnesium ACSF, the postsynaptic response was abolished while the potentials generated by the NM axons remained intact (Fig. 3). Postsynaptic potentials returned when standard ACSF was reperfused.

Contralateral stimulation. Figure $2 D$ illustrates averaged field potentials recorded from NL during stimulation of the contralateral NM. A similar evoked response was obtained when the crossed dorsal cochlear tract was stimulated. Again, low-calcium/high-magnesium ACSF reversibly abolished the postsynaptic responses. The postsynaptic field evoked by contralateral stimulation was always a negative-going wave, whereas the field in response to ipsilateral stimulation often showed an early positivity. This is likely due to differences of electrode position relative to the current sources and sinks created during the two modes of stimulation.

Measurements of latencies were obtained from averaged field potentials such as those displayed in Figure 2. These averaged field potentials are representative of field potentials recorded after a single shock stimulus except that averaging reduced the noise in the signal. Figure 4 shows eight individual field potential traces after the contralateral input to NL was stimulated. For visual purposes, these potentials have been displaced vertically. Importantly, the negative peaks for both the presynaptic and postsynaptic responses occur at nearly the same time in every 


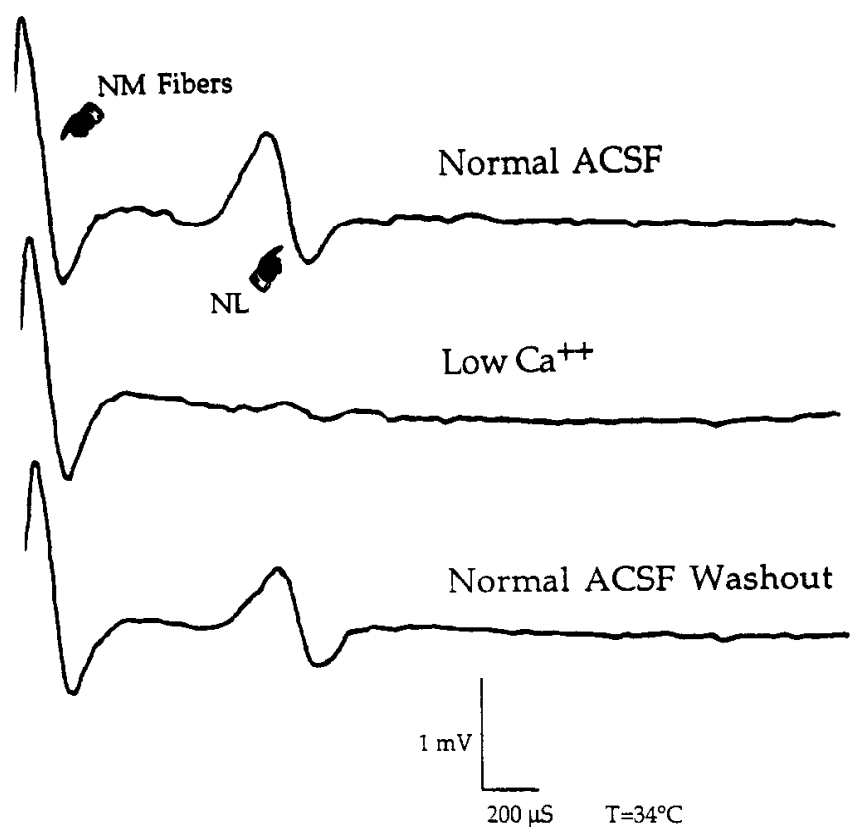

Figure 3. Field potentials recorded in NL during stimulation of ipsilateral NM. Normal ACSF, Recording from NL when the slice was maintained in normal ACSF demonstrating the potentials corresponding to the NM fibers and postsynaptic response of NL neurons. Low $\mathrm{Ca}^{++}$, Recording from NL 4 min after the perfusion medium was changed to one containing low $\mathrm{Ca}^{2+}$ and high $\mathrm{Mg}^{2+}$ concentrations, showing persistence of the NM fiber response but no postsynaptic response. Normal ACSF Washout, Recording from NL 4 min after the slice was reperfused with normal ACSF demonstrating a return of the postsynaptic response.

sweep. The range of the "jitter" in the latency to the postsynaptic response in this case was approximately $20 \mu \mathrm{sec}$.

\section{NL field potentials in different locations within NL}

Ipsilateral stimulation. With the stimulating electrode placed on either the ipsilateral auditory nerve (Fig. $2 A$ ) or ipsilateral $\mathrm{NM}$, field potentials were recorded along the medial-to-lateral course of NL. Figure $2 B$ shows representative recordings obtained when the ipsilateral NM was stimulated. These two recordings were obtained simultaneously with one recording electrode located medially and the other laterally in NL. There was little difference $(10 \mu \mathrm{sec})$ in the latencies of the postsynaptic potentials. Figure 5 illustrates scatter plots of the latencies of postsynaptic potentials as a function of position of the recording electrode within NL from four representative slices. In each case, the latencies did not vary consistently with position $\left(r^{2}=0.00\right.$ 0.28 ). These regressions were not statistically reliable, although variability around the best-fit line was not unusually large (standard error of estimates, $0.02-0.034$ ). Thus, when the ipsilateral $\mathrm{NM}$ was stimulated, the latency of the postsynaptic response across the medial-to-lateral extent of NL was essentially constant.

Contralateral stimulation. With the stimulating electrode placed either on the contralateral NM (Fig. 2C) or on the fibers of the crossed dorsal cochlear tract, field potentials were recorded along the medial-to-lateral course of NL. Figure $2 D$ shows representative recordings obtained when the contralateral NM was stimulated. In these simultaneous recordings, the latency of the postsynaptic response recorded at the laterally placed

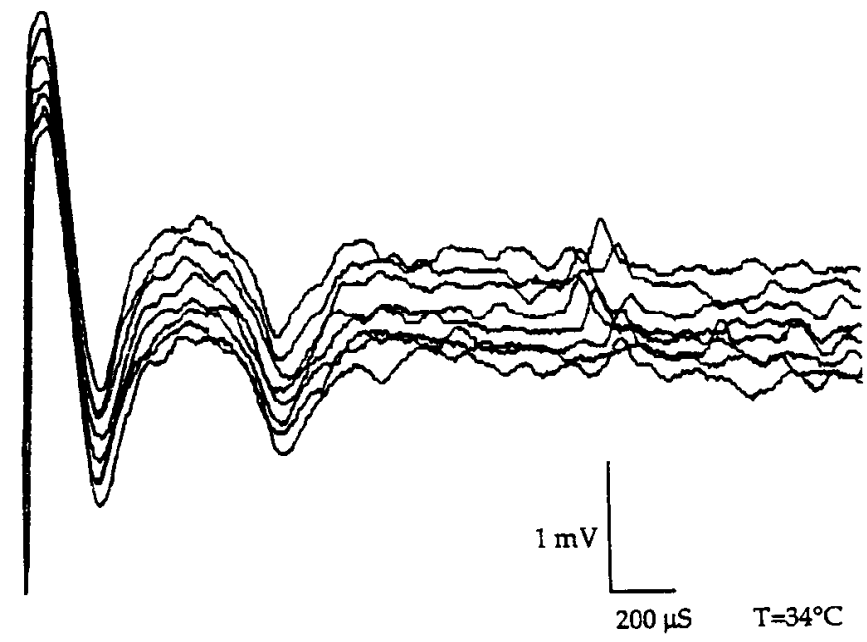

Figure 4. Single pulse field potentials after stimulation of the contralateral projection to NL. The eight single sweeps are slightly displaced from one another vertically in order to aid visualization. The negative peaks of both the pre- and postsynaptic responses occur with approximately the same latency in each sweep. Thus, the averaged field potentials used for the latency measurements presented in the subsequent figures are indicative of individual sweeps.

electrode was always longer than that recorded medially. Scatter plots from four representative slices in which the latencics of postsynaptic responses were measured at different locations in NL are displayed in Figure 6. The $r^{2}$ values for the regression lines ranged from 0.81 to 0.97 ( $p<0.01$ in all cases; standard error of estimate, $0.022-0.034$ ). Thus, when the contralateral input to NL was stimulated, there was a nearly linear increase in the latency of the postsynaptic responses as the recording electrode was moved from medial to lateral positions along NL.

\section{Contralateral NM axon conduction velocity}

Since axons from the contralateral NM course along the ventral surface of NL, conduction velocities of these fibers can be estimated by recording the latencies of the presynaptic field potentials at different positions within NL. All conduction velocities reported in this section are from slices maintained at $34^{\circ} \mathrm{C}$. Figure 7 illustrates scatter plots of the presynaptic field potential latency as a function of distance along NL for four slices. The $r^{2}$ values for these regression lines varied from 0.90 to $0.98(p$ $<0.01$ in all cases; standard error of estimate, 0.012-0.029). The calculated conduction velocities of these axons varied from 2.46 to $4.52 \mathrm{~m} / \mathrm{sec}$. The strong linear fit of the data suggests that fiber velocity does not change dramatically from medial to lateral within NL. Further, the similarity of the slopes in Figures 6 and 7 suggests that the changes in latency of the presynaptic component can account for the majority of the observed changes in the latency of the postsynaptic responses.

Conduction velocity could not be approximated for ipsilateral NM axons. These axons circle around NM and then send terminals out along the dorsal surface of NL (see Fig. 2A). Thus, it was not possible to position recording electrodes at measurable distances along these axons.

\section{Effect of temperature on axon conduction velocity}

To obtain a closer estimate of the magnitude of the delay across NL under physiological conditions, the temperature of the medium was raised toward physiological temperature. Above $36^{\circ} \mathrm{C}$ 
A

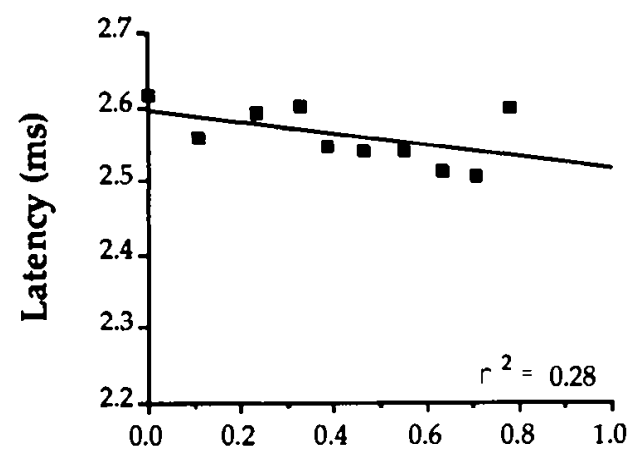

C

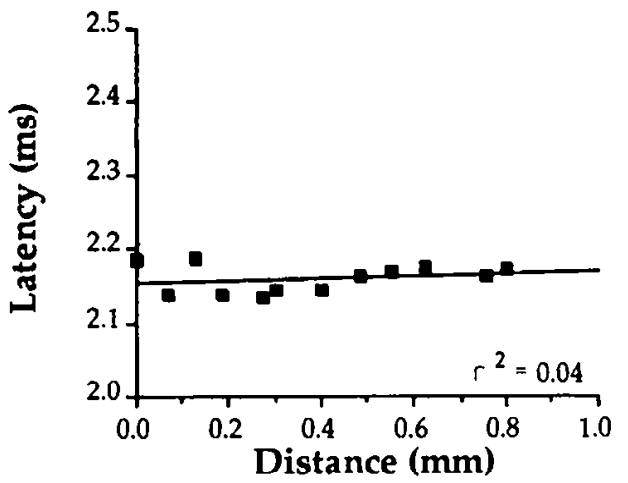

A

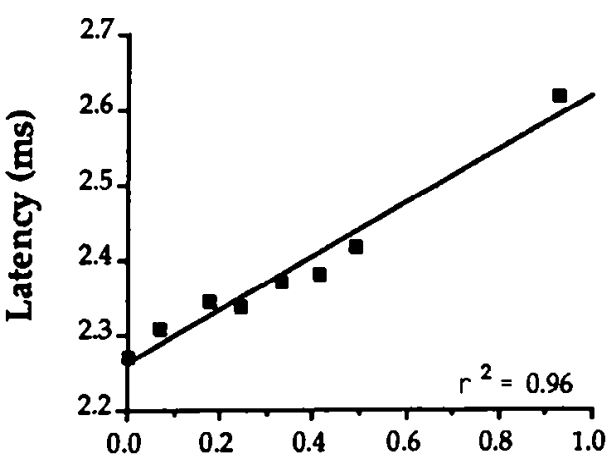

C

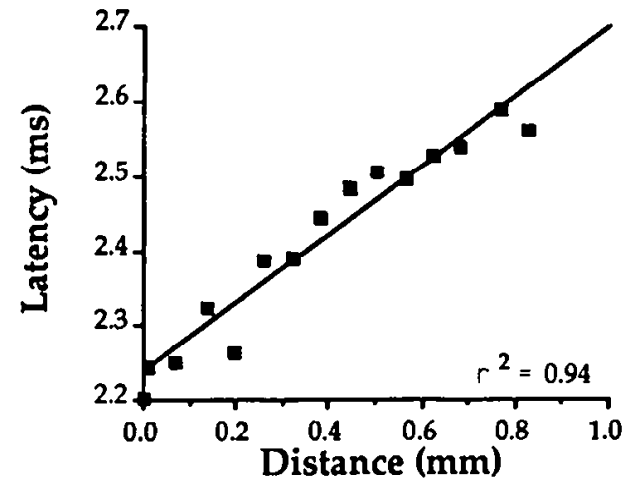

B

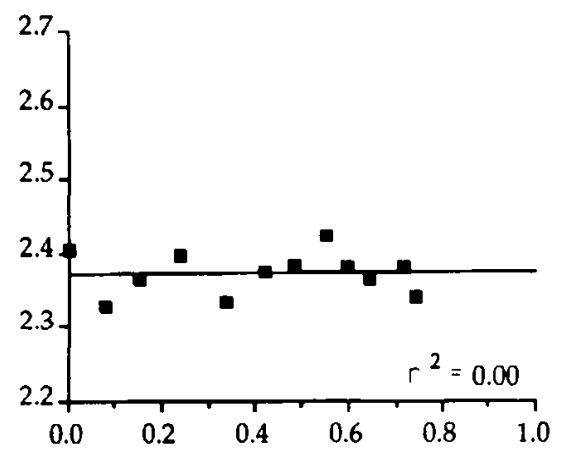

D

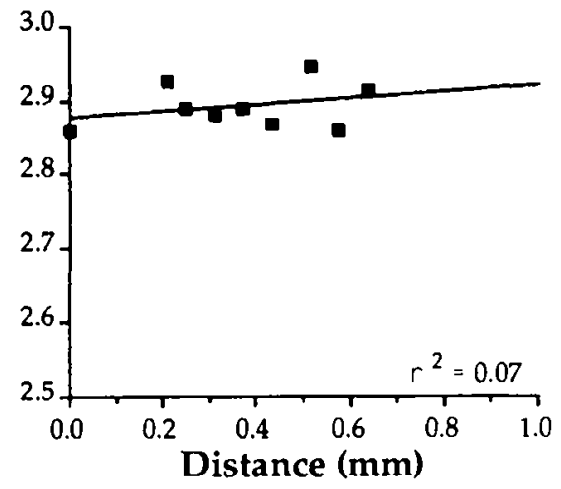

B

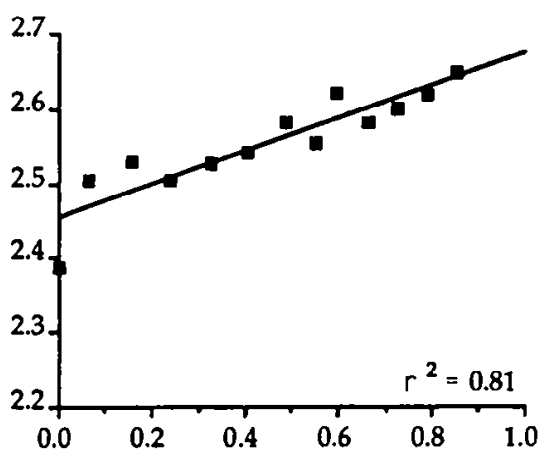

D

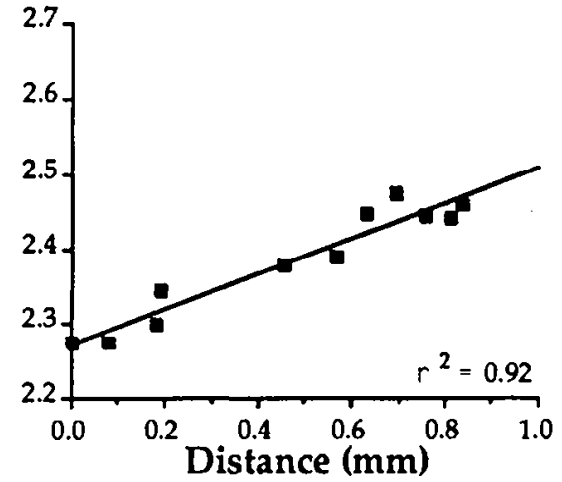

Figure 5. Ipsilateral stimulation: latency of postsynaptic potentials as a function of distance along NL when the stimulus was applicd to the ipsilateral NM $(A-C)$ or ipsilateral auditory nerve $(D)$. Note the low correlation between position and latency of the postsynaptic response. Least-squares regression lines are included. Equations for the regression lines are as follows: $A, y=2.59-$ $0.08 x ; B, y=2.37+0.0015 x ; C, y=$ $2.16+0.014 x ; D, y=2.88+0.04 x$.

Figure 6. Contralateral stimulation: latency of postsynaptic potentials as a function of distance along NL when the stimulus was applied to the contralateral NM $(A)$ or NM axons in the crossed dorsal cochlear tract $(B-D)$. Note the high correlation between position and latency of the postsynaptic response. $A$ and $B$ are data obtained from the slices in which ipsilateral stimulation yielded the data displayed in Figure S, $A$ and $B$. Equations for the least squares regression lines are as follows: $A, y=2.26$ $-0.35 x ; B, y=2.46+0.22 x ; C, y=$ $2.24+0.27 x ; D, y=2.27+0.22 x$. 
A
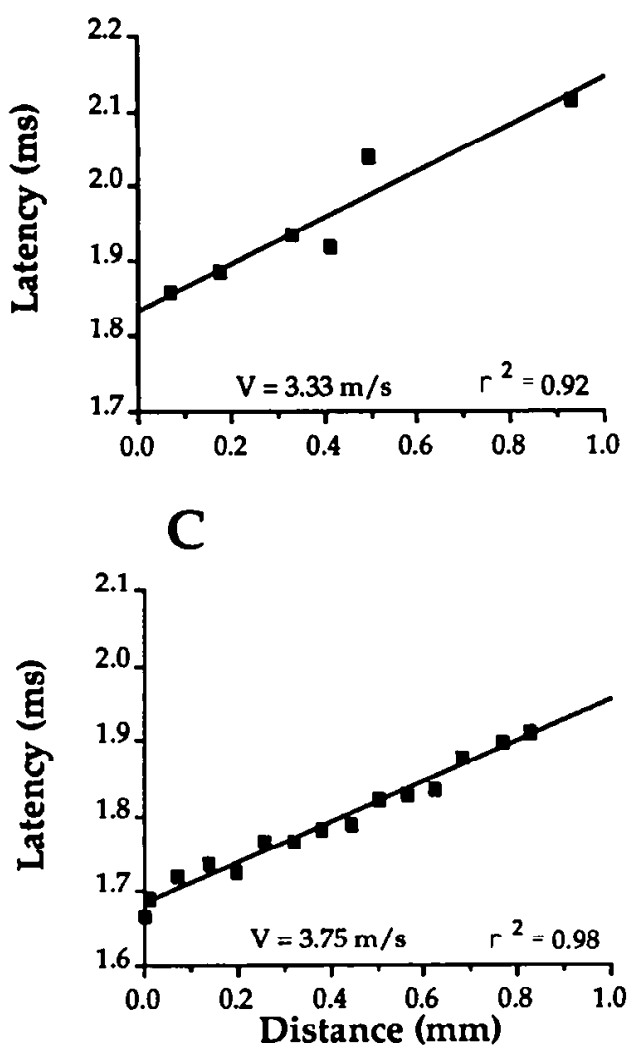

B

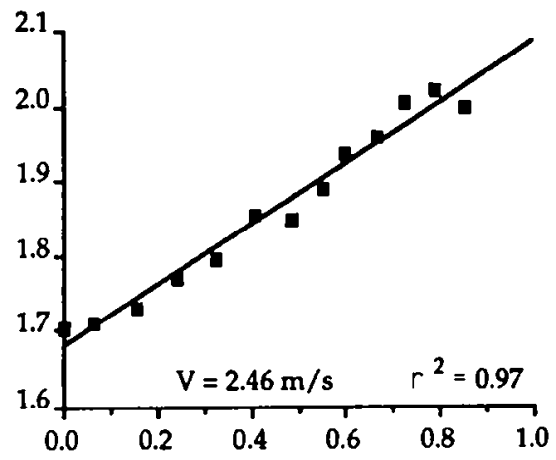

D

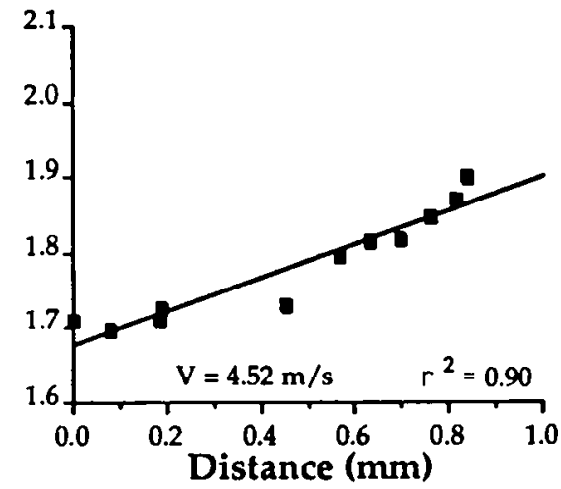

Figure 7. Contralateral NM axon conduction velocity: latency of the NM axon field potential as a function of distance along NL when the stimulus was applied to the contralateral NM $(A)$ or axons in the crossed dorsal cochlear tract $(B-D)$. These data were obtained from the same slices as depicted in Figure 6. The velocity ( $V$ ) across NL is calculated by the reciprocal of the slope of leastsquares regression line. All data were recorded at $34^{\circ} \mathrm{C}$. Equations for the leastsquares regression lines are as follows: $A, y=1.84-0.30 x ; B, y=1.68+$ $0.41 x ; C, y=1.69+0.27 x ; D, y=1.68$ $+0.22 x$. the postsynaptic field potentials became broader and lower in amplitude. These findings are consistent with previously described temperature effects on field potential amplitude obscrved in rabbit hippocampal slices (Shen and Schwartzkroin, 1988). Degradation of field potentials at higher temperatures rendered analysis of the postsynaptic responses at physiological temperature $\left(40-41^{\circ} \mathrm{C}\right)$ impossible. Presynaptic potentials generated by the afferent (NM) fibers, however, were maintained up to and beyond $40^{\circ} \mathrm{C}$ and permitted reliable calculation of fiber velocity at physiological temperature. Figure 8 illustrates the effect of temperature on axon conduction velocity mcasured in two representative slices. Simultaneous recordings from two locations within NL were obtained as the temperature was slowly increased. Fiber conduction velocity increased as the tem- perature of the perfusate was raised. Values of $r^{2}$ from the leastsquare regressions of fiber velocity on temperature ranged from 0.57 to 0.98 ( $p<0.01$ in all cases). The estimates of the fiber velocity at $40^{\circ} \mathrm{C}$ (approximate chick core temperature) ranged from $3.0 \mathrm{~m} / \mathrm{sec}$ to $8.8 \mathrm{~m} / \mathrm{sec}$ with a mean of $5.6 \mathrm{~m} / \mathrm{sec}(n=6$ slices).

\section{Coincidence detection}

To examine the ability of bilateral stimulation to augment the unilateral responses, the intensities of each unilateral stimulating electrode were adjusted to produce little or no discernable postsynaptic field potential when presented alone. The temporal relationship of the ipsilateral and contralateral stimuli was then adjusted such that the postsynaptic fields were coincident. As
Figure 8. Effect of temperature on conduction velocity: conduction velocity of the NM axons as a function of temperature. Simultaneous recordings from a medial and a lateral location in NL were obtained as the temperature of the perfusate increased. The estimate of NM axon conduction velocity at physiological temperature was calculated as the intercept of the least-squares regression line at $T=40^{\circ} \mathrm{C}$. Equations for the regression lines are as follows: $A, y=-8.50+0.35 x ; B, y=1.45+$ $0.038 x$.
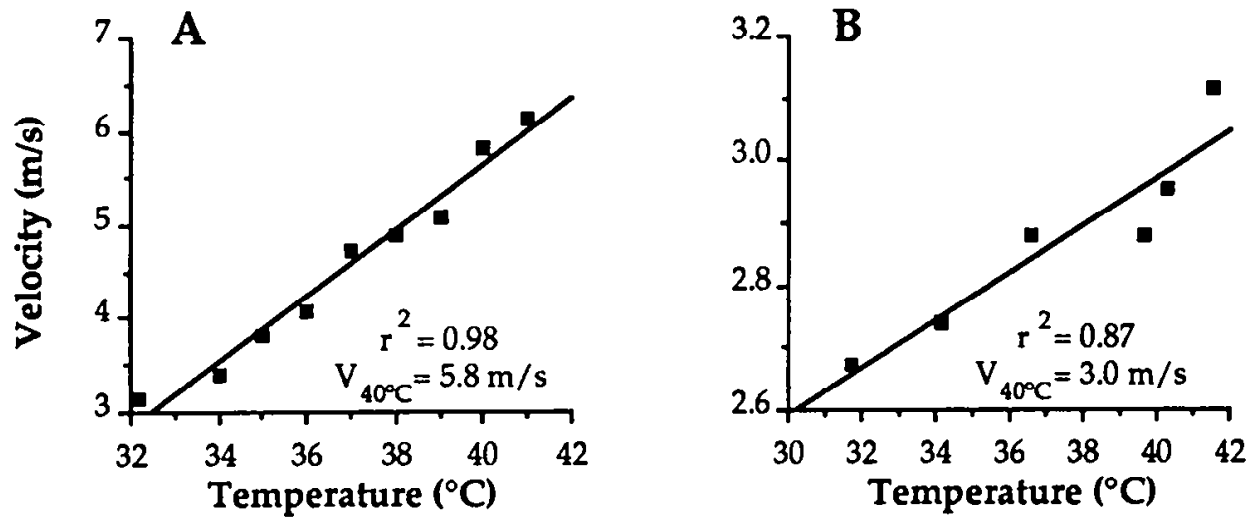


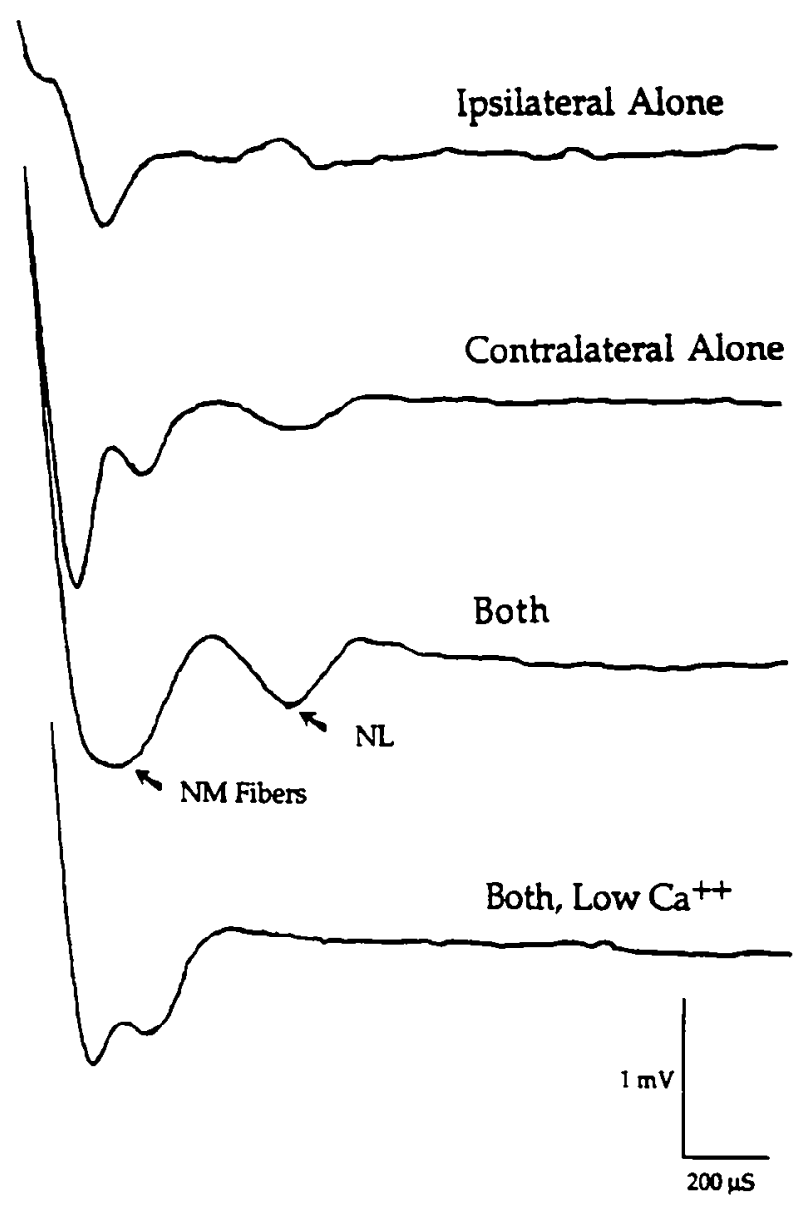

Figure 9. Coincidence of detection in NL: field potentials recorded in NL when the stimulus was applied to the ipsilateral NM alone, the contralateral projection to NL alone (crossed dorsal cochlear tract), or both ipsilateral and contralateral inputs. Note the pronounced postsynaptic response when both inputs to NL were stimulated. The postsynaptic field evoked by bilateral stimulation was abolished when the medium was replaced with one containing low $\mathrm{Ca}^{2+}$ and high $\mathrm{Mg}^{2+}$ concentrations (Both, Low Ca+r ). demonstrated in Figure 9, this procedure produced a pronounced augmentation of the NL response. This was true even though unilateral ipsilateral stimulation often produced a field of polarity opposite to that evoked by contralateral stimulation alone. The amplitude of the postsynaptic field potential evoked by bilateral stimulation is substantially greater than any simple addition of the two unilateral evoked fields. This suggests that NL neurons are integrating subthreshold inputs from each side to produce a large response. As shown in Figure 9, this integration of two subthreshold inputs required the presence of calcium in the medium. This indicates that the evoked field potential is generated by postsynaptic NL neurons.

\section{Mapping of best delay}

In all cases, the amplitude of the postsynaptic field evoked by bilateral stimulation was dependent on the relative timing of stimulating the two inputs. The maximum field was evoked when the timing of the two inputs was adjusted so as to activate the postsynaptic cells simultaneously. Since the contralateral input takes longer to arrive at more lateral locations of NL, optimal time differences must be mapped such that areas in the medial portion of NL would respond maximally to bilateral stimulation having relatively short contralateral lead times, whereas more lateral areas would require a larger contralateral lead to evoke the maximal response. This type of mapping was demonstrated by recording simultaneously from two different areas of NL (see Fig. 10). When the contralateral input (crossed dorsal cochlear tract) was stimulated $200 \mu \mathrm{sec}$ before the ipsilateral NM input (Fig. $10,-200 \mu \mathrm{S}$ ), the postsynaptic field recorded at the medially placed electrode was substantially larger than that recorded at the laterally placed electrode. On the other hand, when the contralateral input was stimulated 100 $\mu$ sec after the ipsilateral input $(+100 \mu \mathrm{S})$, the laterally placed electrode shows the larger response. The peak-to-peak amplitude of the postsynaptic field potentials as a function of the relative timing of stimulation are shown in Figure 11.

\section{Discussion}

It is clear that the brainstem auditory system performs the initial processing of interaural time differences. We have documented

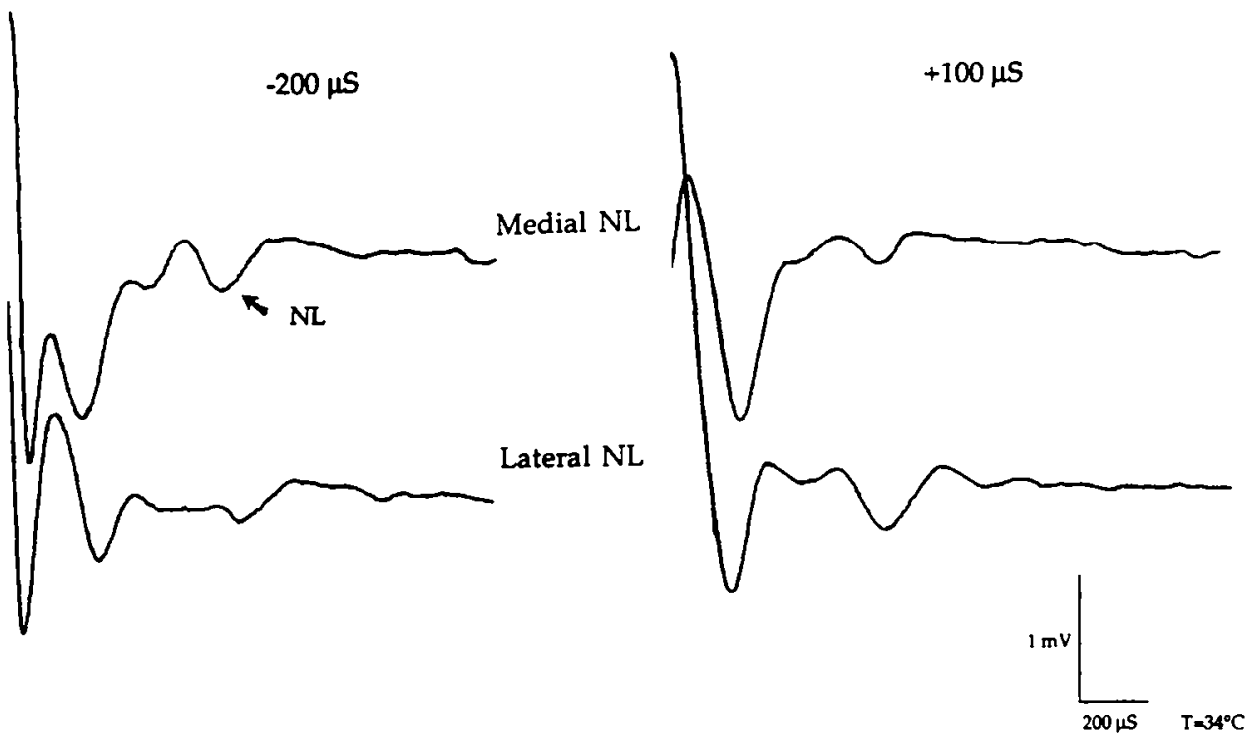

Figure 10. Field potentials recorded simultaneously in medial and lateral locations of NL when both the ipsilateral (NM) and contralateral (crossed dorsal cochlear tract) inputs were stimulated. When the contralateral input was stimulated $200 \mu \mathrm{sec}$ before the ipsilateral input $(-200 \mu \mathrm{S})$, the postsynaptic field recorded in medial NL was larger than that recorded in lateral NL. On the other hand, when the contralateral input was stimulated $100 \mu \mathrm{sec}$ after the ipsilateral input $(+100 \mu S)$, the larger response was recorded in lateral $\mathrm{NL}$. 


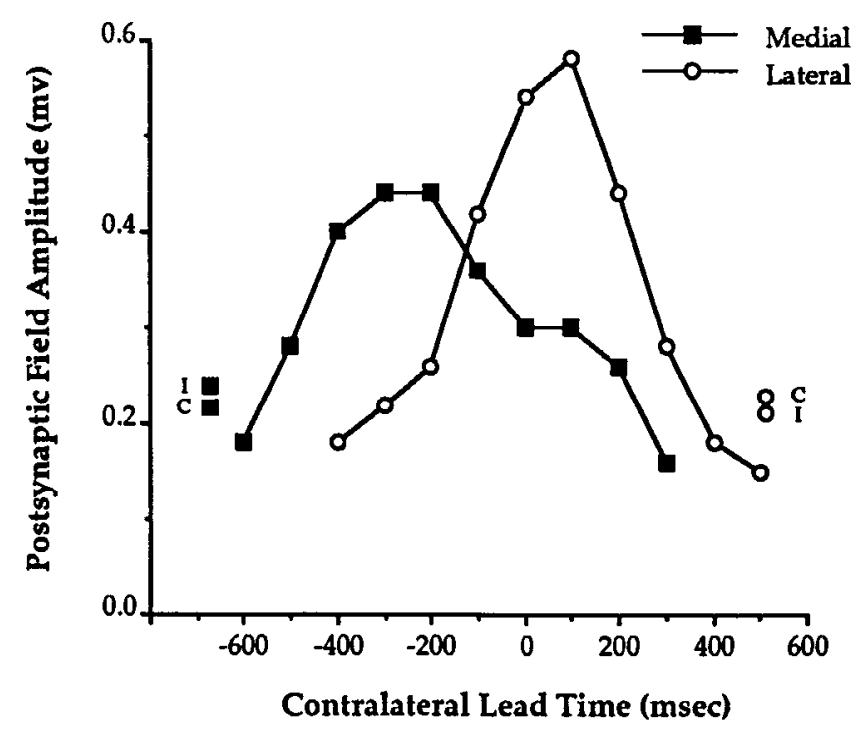

Figure 11. Mapping of optimal time differences: the amplitude of the postsynaptic field potential at two simultaneously recorded positions in $\mathrm{NL}$ is displayed as a function of time delay. The maximal response at the medially placed electrode required a shorter contralateral lead time. Points marked $I$ and $C$ indicate the amplitudes of the postsynaptic fields obtained when the ipsilateral and contralateral inputs were stimulated separately.

two important features of this system in the chick: (1) a delay line from NM across NL and (2) coincidence detection by NL neurons. The combination of these two features results in a map of interaural time delays along the medial-to-lateral extent of the nucleus. Below, we will first discuss some methodological considerations and then describe how these results fit into a general model of coding of interaural time disparities.

\section{Methodological considerations}

This study examined the central pathways between the secondand third-order auditory neurons using an in vitro slice preparation of the chick brainstem. Although a few investigators have successfully recorded from NL (Carr and Konishi, 1990) and the mammalian medial superior olivary nucleus in vivo (Galambos et al., 1959; Moushegian et al., 1964, 1975; Goldberg and Brown, 1968, 1969; Guinan et al., 1972; Crow et al., 1978; Yin and Chan, 1988, 1990), these recordings are reportedly difficult to obtain, primarily due to the relatively small size of the nucleus, the high levels of spontaneous activity, and a large neurophonic response. For the purpose of describing the "delay line" circuit, the brain slice preparation has three key advantages. First, since there is no spontaneous activity in the auditory system in vitro, this preparation allows complete control of afferent (NM) activity, thereby enabling one to track the timing of information as it progresses across NL. Second, in this preparation the stimulating and recording electrodes can be reliably placed under direct visualization and can be efficiently relocated when necessary. This obviates the need for reliance on histological documentation of recording electrode position. Finally, the postsynaptic response can be easily dissociated from the presynaptic response.

Although the slice preparation is a more efficient method for examining the "delay line" circuit in NL, brain slice techniques also have several intrinsic limitations. One limitation relevant to the present analysis is that the best postsynaptic potentials were obtained at relatively low temperatures $\left(34-35^{\circ} \mathrm{C}\right.$ as compared to $40-41^{\circ} \mathrm{C}$ physiological temperature). Although the latency to postsynaptic activation is the crucial aspect for modeling this circuit, analyses at relatively low temperatures revealed that changes in the latency of the postsynaptic field in NL mirrors the variation in the latency of the presynaptic input (i.e., there is no consistent variation in the synaptic delay across NL). Thus, below we use the measurements of the presynaptic component at physiological temperature to estimate the length of the delay line across NL.

A second limitation of the in vitro preparation relates to the plane in which the brainstem slice was sectioned. NL is arranged in a series of isofrequency bands that run orthogonal to the tonotopic axis. The tonotopic axis maps high frequencies rostromedially and lower frequencies caudolaterally (Rubel and Parks, 1975). Any given slice is not likely to have been exactly parallel to the isofrequency bands. Thus, it is possible that different populations of fibers were recorded from medial as compared to lateral positions in NL. Furthermore, the plane of section varies between slices. This variability may result in slight inaccuracies in estimating the total length of the "delay line," but should not alter conclusions about general trends observed in the data.

Finally, in the slice preparation, it is not possible to define accurately the best frequency of the neurons. Most slices probably contained the low- to mid-frequency portions of NL since this is the portion of the nucleus that overlaps with NM in the coronal plane. Because the exact frequencies represented in the slice are not known, it is impossible to discuss our analyses of interaural time differences in terms of interaural phase differences.

\section{A brainstem circuit for binaural processing}

The existence of a brainstem circuit that could analyze time differences of sound reaching the two ears was hypothesized based on previous anatomical studies (Parks and Rubel, 1975; Young and Rubel, 1983, 1986). NM neurons project bilaterally to NL, with each NM neuron projecting to many cells along an isofrequency band in NL. One major conclusion from the present study is that electrophysiological data are consistent with hypotheses derived from the pattern of NM axonal arborizations; along the medial-lateral extent of NL, impulses from the ipsilateral NM arrive at all points within NL simultaneously while those from the contralateral NM arrive with sequentially increasing delays at successively more lateral positions.

This collection of axonal projections resembles the hypothetical mechanism for localization of low-frequency tones described by Jeffress (1948). Figure $1 B$ is a modification of Jeffress's model representing the chicken brainstem auditory nuclei and projections from NM to NL (Young and Rubel, 1983). In contrast to Jeffress's model, the afferents from the ipsilateral NM have equal lengths instead of progressively longer axonal lengths. The projections from the contralateral NM are similar to those proposed by Jeffress, with the most lateral cell in NL receiving afferent input at a later time than the most medial cell in NL. The primary factor in the delay of neuronal activationfrom caudomedial to rostrolateral NL is the conduction time of the small myelinated axons as they course along the ventral aspect of the nucleus.

As in Jeffress's (1948) model, NM afferents could simultaneously activate a specific population of NL neurons depending on the position of the sound source. The schematic in Figure 1, 
for example, depicts that when a sound source is located at midline, neurons in the medial region of NL will receive coincident stimulation of their dorsal and ventral dendrites. More medial neurons will receive input from the contralateral NM slightly earlier than input from the ipsilateral side and more lateral neurons will receive the contralateral input slightly later. If, however, a sound source is displaced laterally, the NM nearest to the source will be activated earlier and impulses will progress more laterally in the contralateral NL before input from the other NM arrives. Thus, different cells in NL will receive coincident input to their dendrites depending on the location of the sound source. Jeffress proposed that neurons receiving $\mathrm{co}-$ incident input would be maximally activated. The present studies show that the activity of NL neurons does, in fact, depend on whether or not inputs from the two sides arrive at approximately the same time. Preliminary data from intracellular recordings of NL neurons also support this conclusion (Hyson et al., 1989). Further, different time disparities evoke maximal responses at different locations within NL. As predicted by the observed medial-to-lateral delay line produced by the contralateral projection to $\mathrm{NL}$, greater contralateral lead times are required to activate neurons maximally in more lateral locations of NL.

The estimates of the afferent fiber velocities from the contralateral NM indicate that one NL could encode approximately $180 \mu \mathrm{sec}$ of "delay" from its most medial aspect to its most lateral extent. This estimate is calculated from the average conduction velocity measured at physiological temperature $(5.6 \mathrm{~m} /$ $\mathrm{sec}$ ) and using $1 \mathrm{~mm}$ as an approximate length of an NL isofrcquency band. The distance of $1 \mathrm{~mm}$ was determined from the distances measured in slices from which recordings were obtained. Assuming that the maximum delay range that NL can account for is $180 \mu \mathrm{sec}$, it would appear, at first glance, that there is a considerable amount of redundancy within NL since the small head-width of the chicken would provide a considerably smaller interaural delay. The diameter of a 5-15-d-old chicken's head is approximately $2 \mathrm{~cm}$. This would result in a maximum interaural delay of less than $100 \mu \mathrm{sec}$. Most birds have similar small head-widths, which would provide for seemingly small interaural delays. One might argue that these relatively small head-widths make interaural delay an unlikely localization cue for birds. The results of recent investigations, however, suggest that avian interaural delays for low frequencies are substantially longer than predicted by the expected path length around the head. Calford and Piddington (1988) measured the interaural delay in several species of birds by simultaneously recording the cochlear microphonics in both ears for sound sources from midline to $90^{\circ}$ azimuth. For low frequencies, the measured interaural delay was considerably longer than estimated from the path length around the head. In the quail, which has a head size similar to that of the young chicken, the measured interaural delay with a sound source at $90^{\circ}$ azimuth was $125-200 \mu \mathrm{sec}$ for low-frequency tones. This surprisingly long interaural delay is apparently due to an interaural canal between the bird's ears. Thus, even birds with small heads have long interaural delays for low frequencies and may use this cue for localization. In this context, an estimated delay of $180 \mu \mathrm{sec}$ across NL suggests that NL on each side could encode the location of sound sources over a range of approximately $90^{\circ} \mathrm{az}-$ imuth.

The hypothesis that NL analyzes information related to interaural time differences has also been examined in the barn owl (Sullivan and Konishi, 1986; Carr and Konishi, 1988, 1990; Konishi et al., 1988). These analyses suggest that the delay lines are oriented in the dorsoventral axis, with replicates along the medial-lateral axis resulting in optimal delay contours across an isofrequency plane of cells. Medial-to-lateral delay lines could also be of importance in the owl, since variation in axonal length appears to be greatest along the medial-to-lateral axis of the contralateral projection, as opposed to the dorsal-ventral axis through NL (Carr and Konishi, 1988). The isodelay contours reported by Sullivan and Konishi (1986), however, show only a small shift toward longer "optimal" contralateral lead times as more lateral aspects of $\mathrm{NL}$ are examined. In the monolayered NL of the chick, dorsal-to-ventral delay lines obviously cannot exist. Systematic variations in delay were observed along the medial-to-lateral extent of the chick's NL. Correspondingly, a map of optimal delay appears to be oriented along this axis. Although there appear to be some differences between the barn owl's delay line system and that of the chick, both systems support the Jeffress model (or simple modifications of it). Specifically, NM projections function as delay lines and NL neurons function as coincidence detectors.

\section{References}

Boord RL (1969) The anatomy of the avian auditory system. Ann NY Acad Sci 167:186-198.

Calford MB, Piddington RW (1988) Avian interaural canal enhances interaural delay. J Comp Physiol A 162:503-510.

Carr CE, Konishi M (1988) Axonal delay lines for time measurement in the owl's brainstem. Proc Natl Acad Sci USA 85:8311-8315.

Carr CE, Konishi M (1990) A circuit for detection of interaural time differences in the brain stem of the barn owl. J Neurosci 10:32273246.

Colburn HS, Durlach NI (1978) Models of binaural interaction. In: Handbook of perception (Carterette EC, Friedman MP, eds), pp 467518. New York: Academic.

Crow G, Rupert A, Moushegian G (1978) Phase locking in monaural and binaural medullary neurons: implications for binaural phenomena. J Acoust Soc Am 64:493-501.

Galambos R, Schwartzkopff J, Rupcrt A (1959) Microelectrode study of superior olivary nuclei. Am J Physiol 197:527-536.

Goldberg JM, Brown PB (1968) Functional organization of the dog superior olivary complex: an anatomical and electrophysiological study. J Neurophysiol 31:639-656.

Goldberg JM, Brown PB (1969) Response of binaural neurons of dog superior olivary complex to dichotic tonal stimuli: some physiological mechanisms of sound localization. J Neurophysiol 32:613-636.

Guinan JJ Jr, Guinan SS, Norris BE (1972) Single auditory units in the superior olivary complex. I. Responses to sounds and classifications based on physiological properties. Int J Neurosci 4:101-120.

Hackett JT, Jackson H, Rubel EW (1982) Synaptic excitation of the second and third order auditory neurons in the avian brain stem. Neuroscience 7:1455-1469.

Hyson RL, Rubcl EW (1989) Transncuronal regulation of protein synthesis in the brain stem auditory system of the chick requires synaptic activation. J Neurosci 9:2835-2845.

Hyson RL, Overholt EM, Rubel EW (1989) Spatial summation for coding interaural time disparities in nucleus laminaris of the chick. Assoc Res Otolaryngol Abstr 12:34-35.

Jeftress LA (1948) A place theory of sound localization. J Comp Physiol Psychol 41:35-39.

Konishi M, Takahashi TT, Wagner H, Sullivan WE, Carr CE (1988) Neurophysiological and anatomical substrates of sound localization in the owl. In: Auditory function: neurobiological bases of hearing (Edelman GM, Gall WE, Cowan WM, eds), pp 721-746. New York: Wiley.

Moushegian G, Rupert A, Whitcomb MA (1964) Brain-stem neuronal response patterns to monaural and binaural toncs. J Ncurophysiol 27:1174-1191

Moushegian G, Rupert AL, Gidda JS (1975) Functional characteristics of superior olivary neurons to binaural stimuli. J Neurophysiol 38: 1037-1048. 
Oertel D (1983) Synaptic responses and electrical properties of cells in brain slices of the mouse anteroventral cochlear nucleus. J Neurosci 3:2043-2053.

Oertel D (1985) Use of brain slices in the study of the auditory system: spatial and temporal summation of synaptic inputs in cells in the anteroventral cochlear nucleus of the mouse. J Acoust Soc Am 78 328-333.

Parks TN, Rubel EW (1975) Organization and development of brain stem auditory nuclei of the chicken: organization of projections from n. magnocellularis to $n$. laminaris. J Comp Neurol 164:435-448.

Rose JE, Brugge JF, Anderson DJ, Hind JE (1967) Phase locked response to low-frequency tones in single auditory nerve fibers of the squirrel monkey. J Neurophysiol 30:769-793.

Rubel EW, Parks TN (1975) Organization and development of the brain stem auditory nuclei of the chicken: tonotopic organization of n. magnocellularis and n. laminaris. J Comp Neurol 164:41 1-434.

Rubel EW, Smith DJ, Miller LC (1976) Organization and development of brain stem auditory nuclei of the chicken: ontogeny of $n$. magnocellularis and n. laminaris. J Comp Neurol 166:469-490.

Shen K, Schwartzkroin PA (1988) Effects of temperature alterations on population and cellular activities in hippocampal slices from mature and immature rabbit. Brain Res 475:305-316.

Smith DJ, Rubel EW (1979) Organization and development of brain stem auditory nuclei of the chicken: dendritic gradients in nucleus laminaris. J Comp Neurol 186:213-240.

Stevens SS, Newman EB (1936) The localization of actual sources of sound. Am J Psychol 48:297-306.

Sullivan WE, Konishi M (1984) Segregation of stimulus phase and intensity coding in the cochlear nucleus of the barn owl. J Neurosci 4:1787-1799.

Sullivan WE, Konishi M (1986) Neural map of interaural phase difference in the owl's brainstem. Proc Natl Acad Sci USA 83:84008404.

van Bergeijk WA (1962) Variation on a theme of Békésy: a model of binaural interaction. J Acoust Soc Am 34:1431-1437.

von Békésy G (1960) Experiments in hearing, pp 272-313. New York: McGraw-Hill.

Warchol ME, Dallos P (1989) Neural response to very low-frequency sound in the avian cochlear nucleus. J Comp Physiol A 166:83-95.

Warchol ME, Dallos P (1990) Neural coding in the chick cochlear nucleus. J Comp Physiol A 166:721-734.

Winter P, Schwartzkopff J (1961) Form und Zellzahl der akustischen nervenzentren in der medulla oblongato von eulen (Striges). Experientia 17:516-517.

Yin TCT, Chan JCK (1988) Neural mechanisms underlying interaural time sensitivity to tones and noise. In: Auditory function: neurobiological bases of hearing (Edelman GM, Gall WE, Cowan WM, eds), pp 385-430. New York: Wiley.

Yin TCT, Chan JCK (1990) Interaural time sensitivity in medial superior olive of cat. J Neurophysiol 64:465-488.

Young SR, Rubel EW (1983) Frequency-specific projections of individual neurons in chick brain stem auditory nuclei. J Neurosci 3: 1373-1378.

Young SR, Rubel EW (1986) Embryogenesis of arborization pattern and topography of individual axons in $n$. laminaris of the chicken brain stem. J Comp Neurol 254:425-459. 